\title{
On the decay for large $|x|$ of solutions of parabolic equations with unbounded coefficients
}

By

\author{
Takaŝi KusAno***
}

\section{Introduction}

There is much current interest in the Cauchy problem for second order parabolic differential equations with unbounded coefficients:

$$
L u \equiv \sum_{i, j=1}^{n} a_{i j}(x, t) u_{x_{i} x_{j}}+\sum_{i=1}^{n} b_{i}(x, t) u_{x_{i}}+c(x, t) u-u_{t}=f(x, t) .
$$

For example, W. Bodanko [3] has proved the existence and uniqueness of solutions $u(x, t)=0\left(\exp \left(\alpha|x|^{\lambda}\right)\right)$ of the Cauchy problem for (A), assuming that $a_{i j}=0\left(|x|^{2-\lambda}\right), b_{i}=0(|x|)$ and $c=0\left(|x|^{\lambda}\right.$ ) (from above) for large $|x|, \lambda \in(0,2]$. Under similar assumptions D. G. Aronson and P. Besala [1] have constructed a fundamental solution of the equation $L u=0$ and solved the general Cauchy problem for (A) by giving an explicit formula for the solution in terms of the fundamental solution obtained. See also G. N. Smirnova [8]. We also mention a paper by P. Besala and P. Fife [2] in which the asymptotic behavior for large $t$ of solutions of such equations is investigated.

The main purpose of this note is to obtain an information about the behavior of decay for large $|x|$ of solutions of the Cauchy problem containing paraboiic differential operators with unbounded coefficients. It will be shown that an exponential decay property for large $|x|$ of the initial data is preserved for the solutions of the linear

Received August 2, 1967.

Communicated by M. Hukuhara.

* Department of Mathematics, School of Science and Engineering, Waseda University.

** This research was done while the author was a Temporary Member of the Research Institute for Mathematical Sciences, Kyoto University, and was supported in part by Matsunaga Science Foundation. 
homogeneous parabolic equation (A): $L u=0$, provided $a_{i j}=0\left(|x|^{2-\lambda}\right)$, $b_{i}=0(|x|)$ and $c=0(1)$ (from above), $\lambda \in(0,2]$, and also for the nonnegative solutions of the semilinear parabolic equation

$$
\sum_{i, j=1}^{n} a_{i j}(x, t) u_{x_{i} x_{j}}+\sum_{i=1}^{n} b_{i}(x, t) u_{x_{i}}+f(x, t, u)-u_{t}=0,
$$

provided that $a_{i j}=0\left(|x|^{2-\lambda}\right), b_{i}=0(|x|)$ and that the nonlinear term $f(x, t, u)$ is majorized by a concave function $F(t, u)$ with $F(t, 0) \equiv 0$.

The author wishes to thank Professor Masuo Hukuhara for his valuable suggestions.

\section{Statement of results}

We begin by considering the linear homogeneous parabolic equation (A) $(f(x, t) \equiv 0)$. We assume that there exist positive constants $k_{1}, k_{2}, k_{3}$ and $\lambda \in(0,2]$ such that

$$
\begin{aligned}
& 0 \leqq \sum_{i, j=1}^{n} a_{i j}(x, t) \xi_{i} \xi_{j} \leqq k_{1}\left(|x|^{2}+1\right)^{(2-\lambda) / 2} \sum_{i=1}^{n} \xi_{i}^{2}, \\
& \left|b_{0}(x, t)\right| \leqq k_{2}\left(|x|^{2}+1\right)^{1 / 2}(i=1, \cdots, n), \\
& c(x, t) \leqq k_{3}
\end{aligned}
$$

for all $(x, t) \in E^{n} \times[0, T]$ and $\xi=\left(\xi_{1}, \cdots, \xi_{n}\right) \in E^{n}$. We say that a function $w(x, t)$ defined on $E^{n} \times[0, T]$ belongs to class $\boldsymbol{E}^{\lambda}$ for $\lambda \in(0,2]$ if there exist positive constants $\alpha, M$ such that

$$
|w(x, t)| \leqq M \exp \left[\alpha\left(|x|^{2}+1\right)^{\lambda / 2}\right], \quad(x, t) \in E^{n} \times[0, T] .
$$

We prove the following:

Theorem 1. Let $u(x, t)$ be a regular ${ }^{1)}$ solution of (A) belonging to class $\mathbb{E}^{\lambda}$ on $E^{n} \times[0, T]$. If the initial function is such that

$$
|u(x, 0)| \leqq M_{0} \exp \left[-\alpha_{0}\left(|x|^{2}+1\right)^{\lambda / 2}\right], x \in E^{n}
$$

for some positive $\alpha_{0}, M_{0}$, then there exist, for each $t \in(0, T]$, positive numbers $\alpha_{t}, M_{t}$ for which

$$
|u(x, t)| \leqq M_{t} \exp \left[-\alpha_{t}\left(|x|^{2}+1\right)^{\lambda / 2}\right], x \in E^{n} .
$$

1) By a regular solution we mean a function continuous on $E^{n} \times[O, T]$ whose first time derivative and second spatial perivatives are continuous on $\mathrm{E}^{n} \times(\mathrm{O}, \mathrm{T}]$, and which satisfies the given parabolic equation. 
In the Appendix we give an example which shows that in deriving from (2.4) the estimate (2.5) for each $t \in(0, T]$ the assumption (2.3) placed on $c(x, t)$ is in a sense essential and cannot be replaced by a less restrictive one

$$
c(x, t) \leqq k_{3}\left(|x|^{2}+1\right)^{\lambda / 2}
$$

under which the general theory of $\mathbb{E}^{\lambda}$-solutions of (A) is developed.

We now turn to the semilinear parabolic equation (B), for which the conditions (2.1) and (2.2) are assumed to hold.

We assume that there exists a concave function $F(t, u)$ with $F(t, 0)=0$ such that

$$
\sup _{x \in E^{n}} f(x, t, u) \leqq F(t, u), \quad(t, u) \in[0, T] \times E^{1} .
$$

Making use of the device due to I. I. Kolodner and R. N. Pederson [5] we can prove the following:

Theorem 2. Assume that $F, F_{u}, F_{u u}$ are continuous and that $F_{u u} \leqq 0$ on $[0, T] \times E^{1}$. Let $u(x, t)$ be a nonnegative regular solution of (B) belonging to class $\mathbb{E}^{\lambda}$ and satisfying

$$
0 \leqq u(x, 0) \leqq M_{0} \exp \left[-\alpha_{0}\left(|x|^{2}+1\right)^{\lambda / 2}\right], x \in E^{n}
$$

for some positive constants $\alpha_{0}, M_{0}$. Let $u_{0}(x, t)$ be a nonnegative regular solution of the linear homogeneous equation

$$
\sum_{i, j=1}^{n} a_{i j}(x, t) u_{x_{i} x_{j}}+\sum_{i=1}^{n} b_{i}(x, t) u_{x_{i}}-u_{t}=0
$$

satisfying the initial condition $u_{0}(x, 0)=u(x, 0), x \in E^{n}$.

Then, we have

(2.8) $0 \leqq u(x, t) \leqq u_{0}(x, t) \exp \left[\int_{0}^{t} F_{u}(s, 0) d s\right], \quad(x, t) \in E^{n} \times(0, T]$.

This establishes the desired decay property of $u(x, t)$, because, according to Theorem $1, u_{0}(x, t)$ behaves like its initial data for each $t \in(0, T]$.

We note that $\mathrm{H}$. Fujita [4] has obtained a similar result for a class of semilinear parabolic equations of the form (B) but with a different nonlinearity. 


\section{Proofs}

Proof of Theorem 1. Following M. Krzyżański [6] we set

$$
u(x, t)=v(x, t) \exp \left[-\alpha(t)\left(|x|^{2}+1\right)^{\lambda / 2}+\beta(t)\right],
$$

where $\alpha(t)>0$ and $\beta(t)$ are bounded $C^{1}$ functions for $t \geqq 0$ to be specified later. Then, the new dependent variable $v(x, t)$ satisfies the parabolic equation

$$
\sum_{i, j=1}^{n} a_{i j}(x, t) v_{x_{i} x_{j}}+\sum_{i=1}^{n} b_{i}^{*}(x, t) v_{x i}+c^{*}(x, t) v-v_{t}=0
$$

where

$$
\begin{aligned}
b_{i}^{*}(x, t) & =b_{i}(x, t)-2 \lambda \alpha(t) \sum_{j=1}^{n} a_{i j}(x, t) x_{j} \\
c^{*}(x, t) & =c(x, t)+\lambda^{2} \alpha^{2}(t)\left(|x|^{2}+1\right)^{\lambda-2} \sum_{i, j=1}^{n} a_{i j}(x, t) x_{i} x_{j} \\
& -\lambda(\lambda-2) \alpha(t)\left(|x|^{2}+1\right)^{\lambda / 2-2} \sum_{i, j=1}^{n} a_{i j}(x, t) x_{i} x_{j} \\
& -\lambda x(t)\left(|x|^{2}+1\right)^{\lambda / 2-1} \sum_{i=1}^{n}\left[a_{i i}(x, t)+b_{i}(x, t) x_{i}\right] \\
& +\alpha^{\prime}(t)\left(|x|^{2}+1\right)^{\lambda / 2}-\beta^{\prime}(t) .
\end{aligned}
$$

It is clear that there is a number $k_{2}^{*}$, depending on the choice of $\alpha(t)$, such that

$$
\left|b_{\imath}^{*}(x, t)\right| \leqq k_{2}^{*}\left(|x|^{2}+1\right)^{1 / 2}(i=1, \cdots, n) .
$$

In view of $(2.1)-(2.3)$ we have

$$
\begin{aligned}
c^{*}(x, t) \leqq\left(|x|^{2}+1\right)^{\lambda / 2}\left[\alpha^{\prime}(t)\right. & \left.+p \alpha^{2}(t)+q \alpha(t)\right] \\
& +k_{3}+\gamma \alpha(t)-\beta^{\prime}(t),
\end{aligned}
$$

where we have set $p=k_{1} \lambda^{2}, q=k_{2} n \lambda, r=-k_{1} \lambda(\lambda-2)$.

If we define the $C^{1}$ functions by

$$
\alpha(t)= \begin{cases}\frac{\alpha_{0}}{1+p \alpha_{0} t} & (q=0) \\ \frac{q \alpha_{0}}{\left(p \alpha_{0}+q\right) e^{q t}-p \alpha_{0}} & (q>0)\end{cases}
$$




$$
\beta(t)= \begin{cases}k_{3} t+\frac{r}{p} \log \left(1+p \alpha_{0} t\right) & (q=0) \\ k_{3} t-\frac{r}{p q \alpha_{0}^{\prime}} \log \frac{q e^{q t}}{\left(p \alpha_{0}+q\right) e^{q t}-p \alpha_{0}} & (q>0),\end{cases}
$$

and if we note that they satisfy the relations

$$
\alpha^{\prime}(t)+p \alpha^{2}(t)+q \alpha(i)=0, k_{3}+r \alpha(t)-\beta^{\prime}(t)=0,
$$

we have from (3.2)

$$
c^{*}(x, t) \leqq 0,(x, t) \in E^{n} \times[0, T] .
$$

Obviously $v(x, t)$ belongs to class $\mathbb{E}^{\lambda}$ on $E^{n} \times[0, T]$ and satisfies the initial condition

$$
|v(x, 0)|=|u(x, 0)| \exp \left[\alpha_{0}\left(|x|^{2}+1\right)^{\lambda / 2}\right] \leqq M_{0}, x \in E^{n}
$$

(note that $\alpha(0)=\alpha_{0}, \beta(0)=0$ ). Appiying a maximum principle due to W. Bodanko ( $[3]$, Theorem 2), we have $|v(x, t)| \leqq M_{0}$ on $E^{n} \times[0, T]$. Hence, by (3.1), we conclude that

$$
|u(x, t)| \leqq M_{0} \exp \left[-\alpha(t)\left(|x|^{2}+1\right)^{\lambda / 2}+\beta(t)\right],(x, t) \in E^{n} \times[0, T],
$$

from which the desired estimate (2.5) follows: $M_{t}=M_{0} e^{\beta(i)}, \alpha_{t}=\alpha(t)$.

Proof of Theorem 2. We observe first that

$$
F(i, u) \leqq F_{u}(t, 0) u \text { for }(t, u) \in[0, T] \times E^{1},
$$

since $F_{u \Delta} \leqq 0$ and $\mathbb{F}(t, 0) \equiv 0$. Hence, the solution $v(t ; \theta)$ of the ordinary differential equation

$$
v_{t}=\mathbb{F}(t, v), \quad v(0)=\theta>0
$$

is majorized by the solution $w(t ; \theta)$ of the linear ordinary differential equation

$$
w_{t}=F_{u}(t, 0) w, \quad w(0)=\theta
$$

that is,

$$
v(t ; \theta) \leqq w(t ; \theta)=\theta \exp \left[\int_{0}^{t} F_{u}(s, 0) d s\right], t \in(0, T]
$$

We compare the solution $u(x, t)$ under consideration with the function $w(t ; \theta)$, noting that the former satisfies the differential ine- 
quality

$$
\sum_{i, j=1}^{n} a_{i j}(x, t) u_{x_{i} x_{j}}+\sum_{i=1}^{n} b_{i}(x, t) u_{x_{i}}+F(t, u)-u_{t} \geqq 0
$$

and that the latter satisfies the linear parabolic equation

$$
\sum_{i, j=1}^{n} a_{i j}(x, t) w_{x_{i} x_{j}}+\sum_{i=1}^{n} b_{i}(x, t) w_{x_{i}}+F_{u}(t, 0) w-w_{t}=0
$$

Taking $\theta \geqq \max _{x \in E^{n}} u(x, 0)$ and applying a comparison theorem of $\mathrm{W}$. Bodanko ([3], Theorem 4), we obtain

$$
u(x, t) \leqq w(t ; \theta),(x, t) \in E^{n} \times[0, T] .
$$

Hence the solution $u(x, t)$ is bounded on $E^{n} \times[0, T]$, though assumed of class $\mathbb{E}^{\lambda}$.

We now consider the function $\bar{u}(x, t) \equiv v\left(t ; u_{0}(x, t)\right)$, the composition of $v(t ; \theta)$ and $u_{0}(x, t)$. Following closely I. I. Kolodner and R. N. Pederson [5] (p. 358) we see that $\bar{u}(x, t)$ satisfies the differential inequality

$$
\sum_{i, j=1}^{n} a_{i j}(x, i) \bar{u}_{x_{i} x_{j}}+\sum_{i=1}^{n} b_{i}(x, t) \bar{u}_{x_{i}}+F(t, \bar{u})-\bar{u}_{t} \leqq 0 .
$$

Subtracting (3.5) from (3.6) we obtain the differential inequality

$$
\sum_{i, j=1}^{n} a_{i j}(x, t) U_{x_{i} x_{j}}+\sum_{i=1}^{u} b_{i}(x, t) U_{x_{i}}+F_{u}\left(t, u^{*}(x, t)\right) U-U_{t} \leqq 0
$$

satisfied by the difference $U(x, t)=\bar{u}(x, t)-u(x, t)$, where $u^{*}(x, t)$ $\therefore$ lies between $\bar{u}(x, t)$ and $u(x, t)$, and hence is bounded on $E^{n} \times[0, T]$. An application of a theorem of W. Bodanko ([3], Theorem 1) yields the inequality

$$
0 \leqq u(x, t) \leqq \bar{u}(x, t), \quad(x, t) \in E^{n} \times[0, T],
$$

since $\bar{u}(x, 0)=u(x, 0)$ initially. The inequality (2.8) follows immediately from (3.7) and (3.4).

\section{Appendix}

The examples which follow are suggested by M. Krzyżański [6].

Example 1. Consider the particular parabolic equation 


$$
\Delta u+\left(k^{2}|x|^{2}+l\right) u-u_{t}=0 \quad\left(\Delta u=\sum_{i=1}^{n} u_{x_{i} x_{t}}\right),
$$

where $k>0$ and $l$ are constants. The solution of this equation belonging to class $\boldsymbol{E}^{2}$ and satisfying the initial condition

$$
u(x, 0)=\exp \left(-\frac{\alpha}{2}|x|^{2}\right), x \in E^{n} \quad(\alpha>0: \text { a constant })
$$

is given explicitly by the formula

$$
u(x, t)=\int_{E^{n}} V(x, t ; y, 0) \exp \left(-\frac{\alpha}{2}|y|^{2}\right) d y
$$

in terms of the fundamental solution $V(x, t ; y, s)$ constructed by A. Szybiak (see [6] and [7]):

$$
V(x, t ; y, s)=\left[\frac{2 \pi}{k} \sin 2 k(t-s)\right]^{-\frac{n}{2}}
$$

$\times \exp \left[-\frac{k}{2}\left(|x|^{2}+|y|^{2}\right) \cot 2 k(t-s)+k\langle x y\rangle \operatorname{cosec} 2 k(t-s)+l(t-s)\right]$,

where $\langle x y\rangle=\sum_{i=1}^{n} x_{i} y_{i}, x, y \in E, 0<t-s<\frac{\pi}{2 k}$.

An easy computation shows that

$$
\begin{gathered}
u(x, t)=\left[\frac{k}{\alpha \sin 2 k t+k \cos 2 k t}\right]^{n / 2} \exp \left[-\frac{k(\alpha \cos 2 k t-k \sin 2 k t)}{2(\alpha \sin 2 k t+k \cos 2 k t)}|x|^{2}+l t\right], \\
(x, t) \in E^{n} \times\left(0, \frac{\pi}{4 k}\right) .
\end{gathered}
$$

Let $t_{0}=\frac{1}{2 k} \tan ^{-1} \frac{\alpha}{k}$. When $t<t_{d}$, the solution $u(x, t)$ decays exponentially as $|x| \rightarrow \infty$; on the contrary it grows exponentially as $|x| \rightarrow \infty$ when $t_{0}<t<\frac{\pi}{4 k}$.

Example 2. Consider the parabolic equation

$$
\Delta u+\left(-k^{2}|x|^{2}+l\right) u-u_{t}=0,
$$

where $k>0$ and $l$ are constants. We are concerned with the solution of this equation satisfying the initial condition

$$
u(x, 0)=\exp \left(\frac{\alpha}{2}|x|^{2}\right), x \in E^{n},
$$

where $\alpha$ is a positive constant less than $k$. Making use of the fundamental solution constructed by A. Szybiak (see $[6\rfloor$ and $[7\rfloor$ ) 


$$
\begin{aligned}
& W(x, t ; y, s)=\left[\frac{2 \pi}{k} \sinh 2 k(t-s)\right]^{-\frac{n}{2}} \\
& \quad \times \exp \left[-\frac{k}{2}\left(|x|^{2}+|y|^{2}\right) \operatorname{coth} 2 k(t-s)+k\langle x y\rangle\right. \\
& \left.\times(\sinh 2 k(t-s))^{-1}+l(t-s)\right]\left(x, y \in E^{n}, 0<t-s<\infty\right),
\end{aligned}
$$

the solution sought is expressed as

$$
u(x, t)=\int_{E^{n}} W(x, t ; y, 0) \exp \left(\frac{\alpha}{2}|y|^{2}\right) d y .
$$

Proceeding as in Example 1 we have

$$
\begin{aligned}
u(x, t) & =\left[\frac{k}{k \cosh 2 k t-\alpha \sinh 2 k t}\right]^{n / 2} \\
\times \exp & {\left[\frac{k(\alpha \cosh 2 k i-k \sinh 2 k t)}{2(k \cosh 2 k t-\alpha \sinh 2 k t)}|x|^{2}+l t\right],(x, t) \in E^{n} \times(0, \infty) . }
\end{aligned}
$$

Let $t_{0}$ be such that $\tanh 2 k t_{0}=\frac{\alpha}{k}$. Though the solution $u(x, t)$ grows exponentially for large $|x|$ if $t<t_{0}$, it decays exponentially for large $|x|$ if $t>t_{0}$.

\section{REFERENCES}

[1] Aronson, D. G. and P. Besala, Parabolic equations with unbounded coefficients, J. Diff. Eqs. 3 (1967), 1-14.

[2] Besala, P. and P. Fife, The unbounded growth of solutions of linear parabolic differential equations, Ann. Scuola Norm. Sup. Pisa, Ser. III. 20 (1966), 719-732.

[3] Bodanko, W., Sur le problème de Cauchy et les problèmes de Fourier pour les équations paraboliques dans un domaine non borné, Ann. Polon. Math. 18 (1966), 79-9!.

[4] Fujita, H., On the blowing up of the Cauchy problem for $u_{\mathrm{t}}=\Delta u+u^{1+\alpha}$, J. Fac. Sci. Univ. Tokyo, Sec. I, 13 (1966), 109-124.

[5] Kolodner, I.I. and R. N. Pederson, Pointwise bounds for solutions of semilinear paraboilic equations, J. Diff. Eqs. 2 (1966), 353-364.

[6] Krzyżański, M., Evaluations des solutions de l'équation linéaire du type parabolique à coefficients non bornés, Ann. Polon. Math. $\mathbb{1}$ (1962), 253-260.

[7] Krzyżański, M. and A. Szybiak, Construction et étude de la solution fundamentale de l'équation linéaire du type parabolique dont le dernier coefficient est non borné I, II, Atti Accad. Naz. Lincei, Cl. Sc. Fis. Mat. Natur. Ser. VIII, 27 (1959), 1-10.

[8] Smirnova, G.N., The Cauchy problem for parabolic equations degenerating at infinity, Mat. Sb. 70 (112)(1966), 591-604. (Russian) 